\title{
Inhibitory signals converge
}

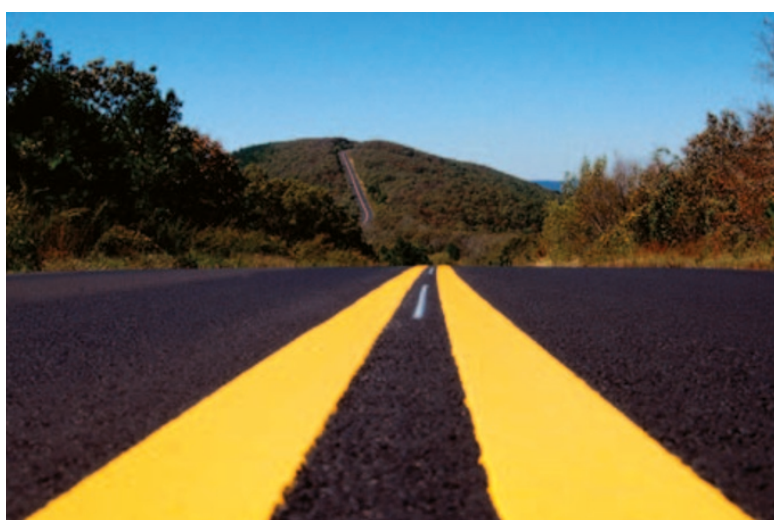

RhoA is a key protein in the intracellular signalling cascades that mediate growth cone collapse and contribute to the failure of axonal regeneration in the injured mammalian CNS. However, blocking RhoA is limited as a therapeutic strategy owing to the numerous roles of this molecule outside the CNS. Fournier and colleagues have now identified another key protein involved in RhoA-mediated neurite outgrowth inhibition that could provide a more specific therapeutic target.

The authors identified the cytosolic phosphoprotein collapsinresponse mediator protein $4 \mathrm{~b}$
(CRMP4b) as a molecule that directly and specifically binds to RhoA. This binding is regulated by the presence of Nogo-66, a potent inhibitory fragment of Nogo-A, one of the key molecules associated with neurite outgrowth inhibition, suggesting that the interaction could be important for suppressing axon outgrowth.

Using rat dorsal root ganglia (DRG) neurons, they showed that RhoA and CRMP4b, which are normally separate within the growth cone, rapidly colocalize at discrete sites when stimulated with myelin. The amino-terminal region of CRMP4b was crucial for this interaction.

The authors demonstrated that knocking down CRMP4b by small interfering RNA in rat DRG neurons enhanced neurite outgrowth when the cells were grown on a myelin substrate. They next introduced a construct (C4RIP-V5) into chick DRG neurons that acted as a competitive antagonist of CRMP4b-RhoA binding. C4RIP-V5 enhanced neurite outgrowth on a myelin substrate, suggesting that CRMP4b-RhoA binding is central to the inhibitory effects of myelin proteins. Furthermore, the construct also attenuated the inhibition of neurite outgrowth by aggrecan, a chondroitin sulphate proteoglycan that also contributes to inhibition of axon outgrowth in the injured CNS.

Fournier and colleagues show that the interaction between CRMP4b and RhoA is a key mediator of growth cone collapse in response to inhibitory substrates. The mechanisms by which CRMP4b induces growth cone collapse have not been determined, but the authors provide evidence that interactions with the actin cytoskeleton might be important. CRMP4b may be a point of convergence for several different inhibitory pathways and could provide a specific target for strategies aiming to enhance axon regeneration after injury.

Katherine Whalley

ORIGINAL RESEARCH PAPER Alabed, Y.Z.,

Pool, M., Ong Tone, S. \& Fournier, A. E.

Identification of CRMP4 as a convergent

regulator of axon outgrowth inhibition.

J. Neurosci. 27, 1702-1711 (2007)

FURTHER READING Yiu, G. \& He, Z. Glial

inhibition of CNS axon regeneration. Nature Rev. Neurosci. 7, 617-627 (2006) 